\title{
The multi-omic landscape of transcription factor inactivation in cancer
}

\author{
Andrew E. Teschendorff ${ }^{1,2,3^{*}}$, Shijie C. Zheng ${ }^{1}$, Andy Feber ${ }^{4}$, Zhen Yang ${ }^{1}$, Stephan Beck ${ }^{4}$ \\ and Martin Widschwendter ${ }^{3}$
}

\begin{abstract}
Background: Hypermethylation of transcription factor promoters bivalently marked in stem cells is a cancer hallmark. However, the biological significance of this observation for carcinogenesis is unclear given that most of these transcription factors are not expressed in any given normal tissue.

Methods: We analysed the dynamics of gene expression between human embryonic stem cells, fetal and adult normal tissue, as well as six different matching cancer types. In addition, we performed an integrative multi-omic analysis of matched DNA methylation, copy number, mutational and transcriptomic data for these six cancer types.

Results: We here demonstrate that bivalently and PRC2 marked transcription factors highly expressed in a normal tissue are more likely to be silenced in the corresponding tumour type compared with non-housekeeping genes that are also highly expressed in the same normal tissue. Integrative multi-omic analysis of matched DNA methylation, copy number, mutational and transcriptomic data for six different matching cancer types reveals that in-cis promoter hypermethylation, and not in-cis genomic loss or genetic mutation, emerges as the predominant mechanism associated with silencing of these transcription factors in cancer. However, we also observe that some silenced bivalently/PRC2 marked transcription factors are more prone to copy number loss than promoter hypermethylation, pointing towards distinct, mutually exclusive inactivation patterns.
\end{abstract}

Conclusions: These data provide statistical evidence that inactivation of cell fate-specifying transcription factors in cancer is an important step in carcinogenesis and that it occurs predominantly through a mechanism associated with promoter hypermethylation.

Abbreviations: BLCA, Bladder carcinoma; CNV, Copy number variation; COAD, Colon adenoma carcinoma; DNAm, DNA methylation; hESC, Human embryonic stem cell; KIRC, Kidney renal cell carcinoma; KIRP, Kidney renal papillary carcinoma; LSCC, Lung squamous cell carcinoma; LUAD, Lung adenoma carcinoma; miRNA, MicroRNA; PRC2, Polycomb repressive complex 2; SCM2, Stem Cell Matrix-2; STAD, Stomach adenocarcinoma; TCGA, The Cancer Genome Atlas; TF, Transcription factor

\section{Background}

Transcription factors (TFs) play a central role in development, specifying differentiation and cell fate [1], as well as in reprogramming [2]. Inactivation of TFs that are important for the specification of a tissue type has been proposed as a key mechanism underlying neoplastic transformation

\footnotetext{
* Correspondence: a.teschendorff@ucl.ac.uk

${ }^{1}$ CAS Key Laboratory of Computational Biology, CAS-MPG Partner Institute for Computational Biology, Chinese Academy of Sciences, Shanghai Institute for Biological Sciences, 320 Yue Yang Road, Shanghai 200031, China

${ }^{2}$ Statistical Cancer Genomics, UCL Cancer Institute, University College

London, Paul O'Gorman Building, 72 Huntley Street, London WC1E 6BT, UK

Full list of author information is available at the end of the article
}

of that tissue [3-7]. Biological evidence for this model has recently come from studies showing how genetic mutations in epigenetic regulators such as isocitrate dehydrogenases can result in the inactivation of key transcription factors, promoting cancer $[8,9]$.

Surprisingly, however, there is a lack of statistical evidence supporting a model in which silencing of transcription factors constitutes a general process underpinning cancer. Arguably, the strongest statistical evidence so far derives from the long-standing observation that bivalently or polycomb repressive complex 2 (PRC2)-marked promoters in human embryonic stem cells (hESCs), which 
often mark transcription factors that are needed for development and differentiation [10,11], are significantly more likely to be hypermethylated in cancer $[4,5,12]$ and aged normal tissue [13-15] compared with random gene sets. However, even though increased promoter methylation is usually associated with gene silencing, the significance of the observed hypermethylation in cancer is unclear because a large proportion of these bivalently or PRC2-marked TFs are not expressed in the corresponding normal tissue type $[16,17]$. Moreover, inactivation of key transcription factors has been associated with other epigenetic alterations such as histone remodelling $[8,9]$, raising further questions as to the role of the observed DNA hypermethylation in cancer. For instance, epigenetic silencing of HNF4A (a key liverspecifying TF) in liver cancer has been linked to loss of promoter H3K4me3 without changes in promoter methylation [8]. Given the large-scale availability of mutational, copy number variation (CNV) and DNA methylation data in primary cancer material, no study has yet systematically explored which mechanism, i.e. mutation, CNV loss, or promoter hypermethylation, is predominantly associated with in-cis silencing of transcription factors in cancer.

The purpose of this study, therefore, is to conduct a detailed exploration of the molecular multi-omic landscape of transcription factor inactivation in cancer. We focus our analysis on a subset of bivalently/PRC2-marked transcription factors expressed in a given normal tissue and which are preferentially silenced in the corresponding cancer type. We point out that this is very different from previous studies, which have largely only reported molecular alteration enrichment patterns (mainly DNA methylation) at either the full repertoire of approximately $1500 \mathrm{TFs}$ or the thousands of genes that are bivalently/PRC2-marked in hESCs $[4,5,12]$. The identification of key bivalently/PRC2marked TFs is achieved by comparing mRNA expression data from hESCs and normal fetal and adult tissues and their corresponding cancer types and studying their patterns of gene expression change across these four phenotypic states. The importance of using normal fetal samples in these types of analyses has recently been highlighted [18], as it allows the confounding effect of age, a major cancer risk factor, to be removed. Having identified the key deregulated TFs in each cancer type, we then perform an integrative multi-omic analysis, encompassing genomewide mRNA expression, DNA methylation, CNV and somatic mutations for six cancer types, revealing that promoter hypermethylation, and not in-cis genomic loss or genetic mutation, is the mechanism that most strongly associates with silencing of these transcription factors in cancer.

\section{Methods}

\section{Definition of initial TF list}

We constructed an initial TF gene list as follows. We first used the definition of human TFs, as defined by the
Molecular Signatures Database from the Broad Institute (http://software.broadinstitute.org/gsea/msigdb/

index.jsp), consisting of a total of 1385 TFs. The most relevant subset of TFs for development and differentiation processes are those which are bivalently or PRC2 marked in hESCs $[10,11]$. This resulted in a list of 458 bivalent/PRC2-marked TFs, of which 403 were also present in the Stem Cell Matrix-2 (SCM2) compendium mRNA expression data set.

\section{The SCM2 compendium data set and identification of TFs expressed in normal tissues}

We downloaded the Illumina mRNA expression data of the SCM2 compendium [19, 20]. Expression data were quantile normalized and probes mapping to the same Entrez gene IDs were averaged. This resulted in an expression data set of 17,967 uniquely annotated Entrez gene IDs and 239 samples, including 107 hESC lines, 52 induced pluripotent stem cells and 32 somatic differentiated tissue samples, with the rest of the samples representing human cell lines. Among the 32 somatic differentiated tissue samples, we selected those epithelial tissues for which there were at least two samples and for which we could identify corresponding cancer data sets from The Cancer Genome Atlas (TCGA). In cases where fetal and adult samples were available, we used fetal samples since these are of age zero, thus eliminating age as a potential confounder [18]. These epithelial tissues included bladder (two adult samples), lung (two fetal samples), kidney (two fetal samples), colon (one fetal and one adult sample) and stomach (three fetal samples). However, the stomach samples were not considered further because the top principal component of variation in the corresponding stomach adenocarcinoma (STAD) TCGA data set correlated with an unknown confounding factor, most likely representing cellular heterogeneity. Thus, for each of the four cell types (lung, kidney, colon and bladder), we derived statistics of differential expression for all 17,967 genes compared with the 107 hESC lines using an Bayes model [21] as implemented in the limma Bioconductor package [22].

\section{TCGA data}

We downloaded TCGA data (as provided by TCGA website), including all level $3 \mathrm{CNV}$, RNA-Seq (V2) and Illumina 450k DNA methylation data, in addition to somatic mutational information, for a total of six cancer types, including lung adenoma carcinoma (LUAD) [23], lung squamous cell carcinoma (LSCC) [24], kidney renal cell carcinoma (KIRC) [25], kidney renal papillary carcinoma (KIRP) [26], bladder carcinoma (BLCA) [27], colon adenoma carcinoma (COAD) [28] and stomach adenomacarcinoma (STAD) [29]. Illumina 450k DNA methylation data were further processed using BMIQ to adjust for the type 2 bias [30]. In the case of RNA-Seq level 3 data, genes 
with zero read counts in all samples or exhibiting no variation across samples were removed. RNA-Seq level 3 data were subsequently regularised using a $\log 2$ transformation. Normalized RNA-Seq and DNA methylation data sets were subjected to an additional quality control procedure which used a singular value decomposition to assess the nature of the top components of variation [31]. According to this analysis, the STAD TCGA dataset was not considered further due to the top component of variation not correlating with normal/cancer status, an indicator of substantial confounding variation [31].

In the case of mutational data, somatic mutations were classed as inactivating mutations if they were nonsense, missense or deletions. For a given tumour sample and gene, multiple inactivating mutations in the same gene were treated as one. In the case of CNV data, we used the normalised segment values as provided by the level 3 standard.

\section{Differential expression and differential DNA methylation analyses}

Differential gene expression analysis for the normalized RNA-Seq data between normal and cancer tissue was performed using an empirical Bayes model [21] as implemented in the limma Bioconductor package [22]. The numbers of normal and cancer samples were 58 and 471 for LUAD, 45 and 473 for LSCC, 72 and 515 for KIRC, 32 and 289 for KIRP, 17 and 323 for BLCA and 41 and 270 for COAD.

In the case of Illumina 450k DNA methylation data we used a recursive model, validated by us previously [32], to assign a DNA methylation (DNAm) level to each gene. Specifically, this model first assigns the average DNAm value of probes mapping to within $200 \mathrm{bp}$ upstream of the transcription start site. If no $450 \mathrm{k}$ probes map to this region, first exon probes are used instead. If there are no first exon $450 \mathrm{k}$ probes for a given gene, we use the average over $450 \mathrm{k}$ probes mapping to within $1500 \mathrm{bp}$ upstream of the transcription start site. As shown by us previously, the average DNAm of $450 \mathrm{k}$ probes in these regions provides the best predictive model of a sample's gene expression value [32]. The same empirical Bayes model was then used to derive statistics of differential DNA methylation between normal and cancer tissue. The numbers of normal and cancer samples for the differential DNAm analysis were 41 and 275 for LSCC, 32 and 399 for LUAD, 160 and 299 for KIRC, 45 and 196 for KIRP, 19 and 204 for BLCA and 38 and 272 for COAD.

\section{Definition of control non-housekeeping gene sets}

In order to objectively assess whether TFs overexpressed in a normal tissue type relative to hESCs exhibit preferential downregulation in the corresponding cancer type, a comparison with a control set of non-housekeeping genes is needed. This control set of genes was constructed for each TCGA cancer set separately as we needed to select genes with similar expression levels to the TFs in the normal-adjacent samples of TCGA set. Having identified a matching set, we then removed all housekeeping genes using the comprehensive list of 3804 housekeeping genes from Eisenberg and Levanon [33]. Thus, the control set of genes consists of nonhousekeeping genes expressed at the same level in normal-adjacent tissue as the given TFs.

\section{Integrative matched tumour analyses}

In order to identify the tumours where a given tissuespecific TF is underexpressed, we derived a $Z$-score for each tumour and TF by comparing its TF expression level with the mean and standard deviation of expression as evaluated over all corresponding normal tissue samples. Specifically, if $t$ labels the TF and $\mu_{t}$ and $\sigma_{t}$ label the mean and standard deviation in expression of this TF over the normal tissue samples, then the $Z$-score of TF $t$ in sample $s$ is defined by $Z_{t s}=\left(X_{t s}-\mu_{t}\right) / \sigma_{t}$. We deemed a TF to be underexpressed in sample $s$ if the corresponding $Z$-score was less than -2 , corresponding to a $P$ value of $\sim 0.05$. For the tumours exhibiting underexpression of the TF, we then defined a genomic loss if the segment value corresponding to the TF locus had a value less than -0.35 (we estimated a conservative threshold of one-copy gain/loss to be at around \pm 0.35 ). For tumours exhibiting underexpression of the TF, we also considered the promoter of the TF to be significantly hypermethylated if the difference in DNA methylation between the tumour and the average of the normal samples was larger than 0.3. This estimate is justified from scatterplots of promoter DNAm versus log2[RNA-Seq counts] for all genes in normal samples, which shows that promoter DNAm increases of 0.3 or higher are much more likely to be associated with gene silencing. In the case of DNAm, an alternative approach could have been to define an analogous $Z$-score of DNAm change in relation to the normal tissue. However, this could generate large statistics without necessarily a big change in absolute DNAm levels; given that the purpose was to see if the DNAm change could account for the change in gene expression, we focused on using absolute differences in DNAm levels.

For the integrative analyses where the matched nature of the samples was used, analysis was restricted to normal and cancer samples with matched DNAm, CNV and mRNA expression data. The numbers of normal and cancer samples for these matched analyses were 8 and 273 for LSCC, 20 and 390 for LUAD, 24 and 292 for KIRC, 21 and 195 for KIRP, 13 and 194 for BLCA and 19 and 253 for COAD. 


\section{Results}

Identification of transcription factors important for tissue differentiation

We posited that TFs with important roles in differentiation and cancer could be identified by analyzing their dynamic expression changes between four main cellular states: the hESC state, a partially differentiated normal fetal state, an adult normal differentiated state and the undifferentiated cancer state. Indeed, as already shown by others in the context of development [1], focusing on dynamic changes in gene expression can successfully identify key TFs. Thus, we initially aimed to identify TFs which become overexpressed in a number of normal tissue types, relative to the hESC ground state, using data from the Stem Cell Matrix-2 (SCM2) compendium [19, 20] ("Methods"). An advantage of using the SCM2 data is the availability of mRNA expression data generated with the same array platform for both hESCs and somatic primary cells for a number of different tissue types, including both fetal and adult states to avoid confounding by age ("Methods"). We restricted the analysis to somatic tissue types for which there were at least two independent samples in the SCM2 compendium and for which there was corresponding high-quality tissue data from TCGA. In total, we identified four tissue types for which matching data in SCM2 and TCGA were available: this included lung, kidney, bladder and colon. Comparison of mRNA expression levels between hESCs (a total of 107 hESC samples derived from both male and females and from a wide range of different passages) and the foetal/adult normal samples from lung, kidney, bladder and colon were performed, focusing on a set of 403 bivalently [10] or H3K27me3 (PRC2) [11] marked TFs in hESCs ("Methods"; Additional file 1: Table S1), since it is well known that their poised promoters in the hESC state mark TFs which are needed for differentiation [10, 11]. We observed that approximately 200 (i.e. 50 \%) of these 403 TFs exhibited significant differential expression relative to the hESC state, a result which was largely independent of tissue type (Fig. 1a). Among the significantly differentially expressed TFs, around 150 (i.e over $70 \%$ ) were overexpressed in the differentiated tissue, supporting their role in differentiation (Fig. 1a, b; Additional file 1: Tables S2-S5). We verified that the overwhelming majority of these significantly overexpressed TFs exhibited fold changes larger than two (Fig. 1c), further supporting their significance. In total, 76 overexpressed TFs were common to all four tissue types, with $19,25,24$ and 18 being overexpressed in only lung, kidney, bladder and colon, respectively (Fig. 1d).

\section{Bivalent/PRC2-marked TFs expressed in a tissue type are preferentially silenced in the corresponding cancer type}

We hypothesized that TFs which are important for differentiation of a tissue type, and which are, therefore, expressed in that tissue type, may be under selection pressure to undergo silencing in the corresponding cancer type. To formally test this, we collected RNA-Seq data from TCGA for two types of lung cancer (LSCC and LUAD), two types of kidney cancer (KIRC and KIRP), BLCA and COAD. In order to draw a statistically valid conclusion in each normal-cancer TCGA dataset, we need to compare the statistics of differential expression of mutually exclusive sets of TFs. Hence, we first focused on the previously identified 19 lung-, 25 kidney-, 24 bladder- and 18 colon-specific TFs, most of which $(18,21,19$ and 14, respectively) were also highly expressed in the respective normal tissue samples from TCGA. In order to assess the biological and statistical significance, the comparison of these sets of TFs was made to a common control set of genes (CTL) expressed at the same level in the normal tissue as the given TFs and which excluded any of 3804 well-established housekeeping genes [33] (Additional file 1: Figure S1). We observed that the great majority of the identified TFs were significantly downregulated in the corresponding cancer type, with the identified TFs more likely to be downregulated in the corresponding cancer type compared with the control set of genes (Fig. 2a; Additional file 1: Tables S6-S9). Thus, the silencing of these TFs in cancer is not merely determined by their relatively high expression levels in the normal tissue since a control set of non-housekeeping genes expressed at the same level in normal tissue (Additional file 1: Figure S1) did not show the same level of downregulation in cancer (Fig. 2a). As expected, the promoters of the silenced TFs were significantly more likely to map to a $\mathrm{CpG}$ island owing to the fact that we initially restricted the analysis to bivalently and PRC2-marked TFs (Additional file 1: Table S10).

Next, we decided to relax the definition of tissuespecific TFs to allow any TF expressed in a given normal tissue regardless of its expression level in other normal tissue types. This more inclusive definition recognizes that cell and tissue types are arranged in a hierarchical developmental tree, as it is well known that TFs important for specification of one tissue type are also important for specification of other tissues. As a concrete example, FOXA1 $(H N F 4 A)$ is a transcription factor important for the specification of the intestine and stomach [34, 35] as well as liver [36] and silencing of HNF4A leads to liver cancer [8]. Similarly, GATA factors such as GATA4 play key roles in the development of the gastrointestinal tract [37-39] as well as in the development of the heart [40], pancreas [41] and liver [42], and so these factors could play tumour-suppressor roles in many different cancer types $[39,43]$. Hence, TFs expressed in multiple normal tissue types can be as important to the development of a specific cancer type than TFs which are expressed only in the corresponding normal tissue type. Thus, on 


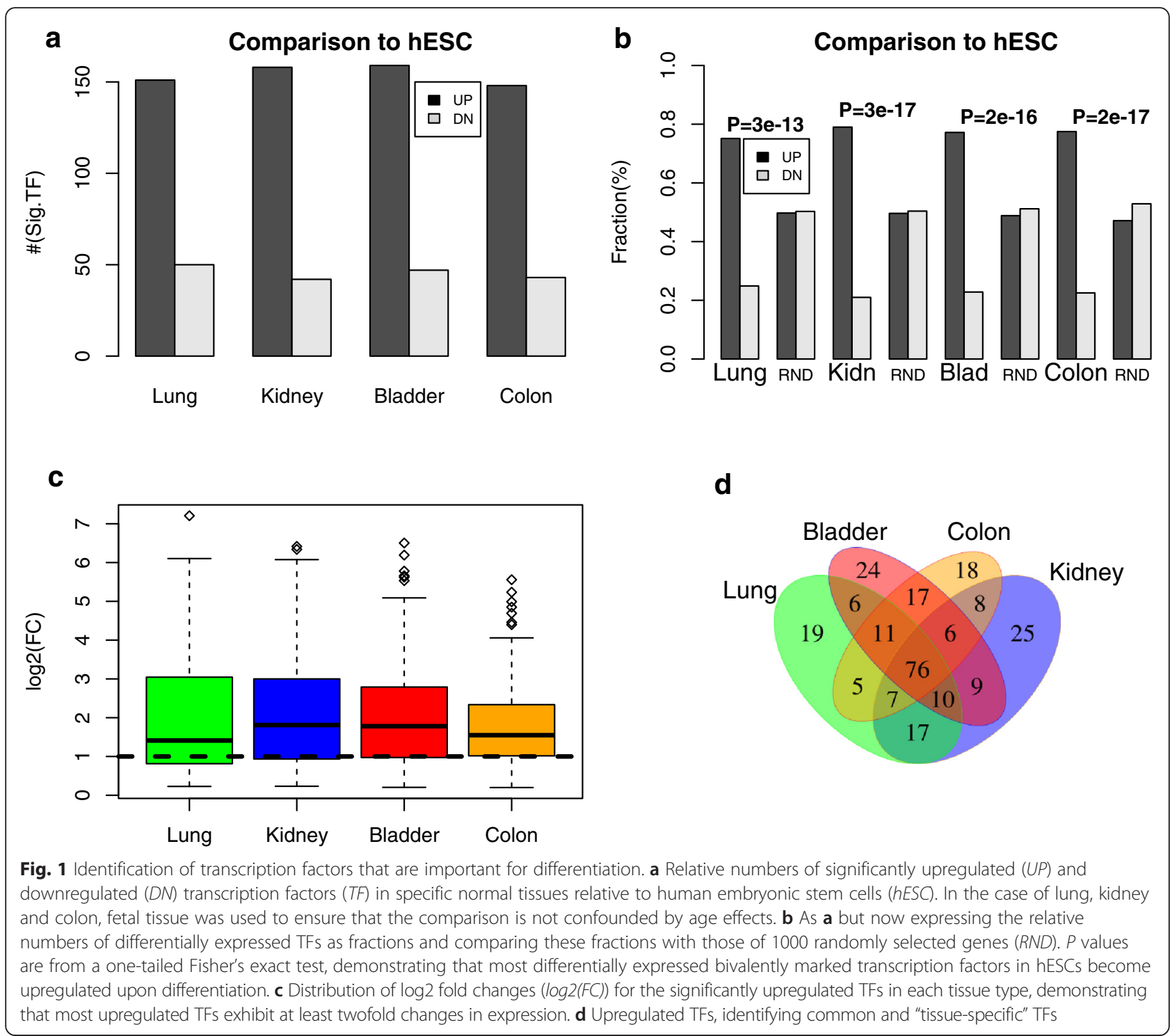

biological grounds, we re-assessed the previous result, now considering all TFs expressed in a normal tissue regardless of their expression levels in the other normal tissues types. In spite of the fact that these TF sets are largely overlapping, we still observed that the strongest underexpression was in the corresponding cancer type and that it was highly significant when compared with a control set of non-housekeeping genes expressed at a similar level in the same normal tissue (Additional file 1: Figures S3 and S4).

Among the silenced TFs were many well known differentiation factors (Fig. 2b). For instance, in lung we found FOXA2 [44], TBX4 [45] and BMP4 [46], and although the role of LHX6 in lung development is less well defined, it has previously been implicated as a tumour suppressor in lung cancer [47]. Similarly, in kidney we observed many TFs implicated in kidney development, including HOX family genes [48], ESRRB/ESRRG [49], PAX2 and LHX1 [50, 51]. In the case of bladder cancer, TFs which have been previously implicated in urothelial cell differentiation, such as RARA and KLF4 [52], were observed to be upregulated in bladder tissue compared with hESCs (Additional file 1: Table S4) and also subsequently silenced in bladder cancer (Additional file 1: Figure S2), although they were also observed to be upregulated in kidney or lung tissue (Additional file 1: Tables S2 and S3). In the case of colon cancer, silenced TFs included well known intestinal differentiation factors such as CDX1 [53, 54], CDX2 [55, 56] and NEUROD1 [57, 58]. Thus, our approach successfully identifies TFs silenced in cancer and which have been previously implicated in the differentiation of the corresponding tissue types. 


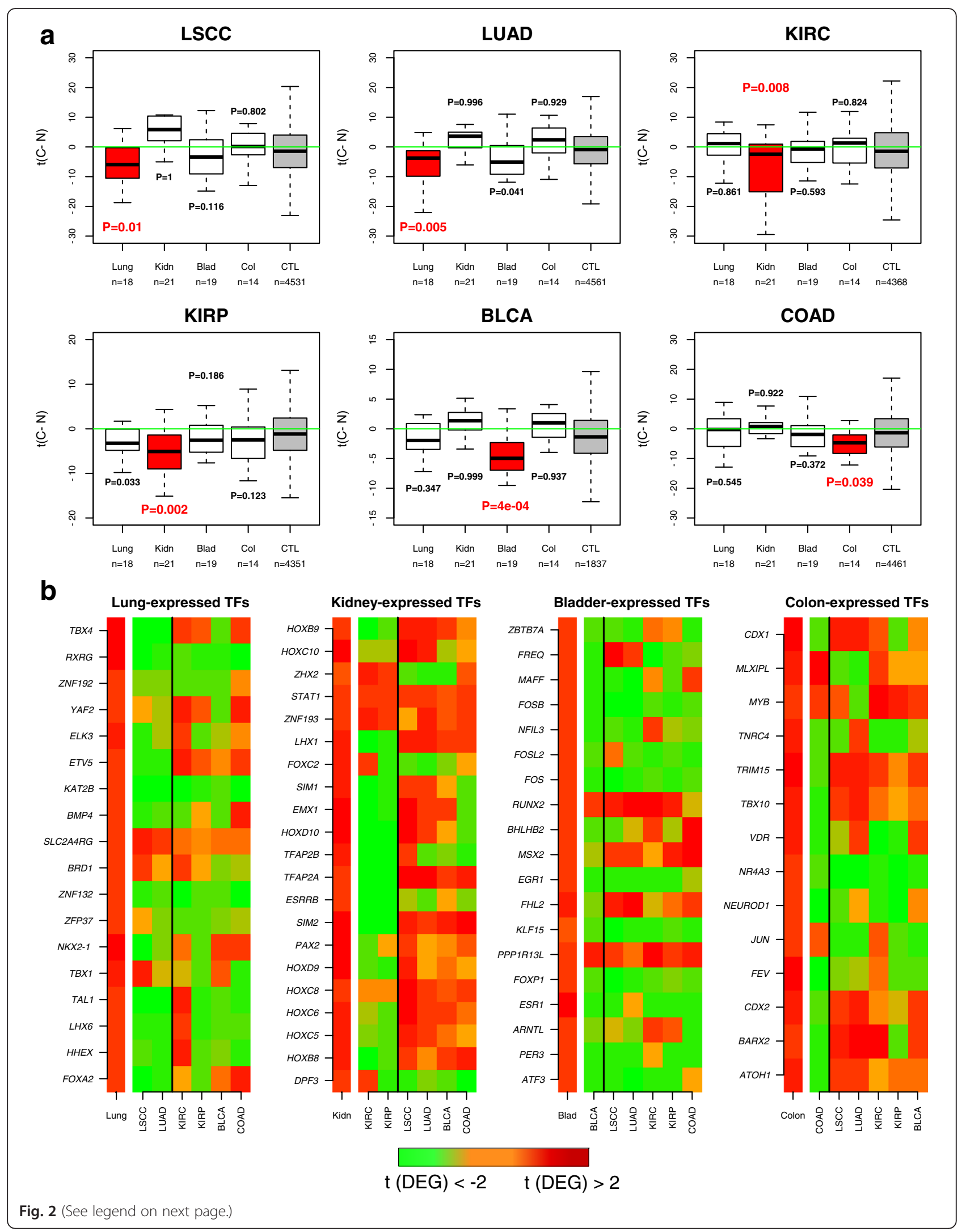




\section{(See figure on previous page.)}

Fig. 2 Transcription factors expressed in normal tissue are preferentially silenced in the corresponding cancer type. a Boxplots of t-statistics of differential mRNA expression between cancer and normal tissue $(y$-axis, $t(C-N))$ for four sets of "tissue-specific" TFs and a control set of genes (CTL) across six different cancer types, as indicated. LSCC lung squamous cell carcinoma, LUAD lung adenoma carcinoma, KIRC kidney renal clear cell carcinoma, KIRP kidney renal papillary carcinoma, BLCA bladder carcinoma, COAD colon adenoma carcinoma. The five sets of genes being compared are the TFs expressed in the relevant normal tissue (red box), the TFs expressed in other normal tissue types (white boxes) and a set of control (CTL, grey box) non-housekeeping genes which are expressed at a similar level to the TFs expressed in that same normal tissue. $P$ values are from a one-tailed Wilcoxon-rank sum test comparing the t-statistics of each group of TFs with the control (CTL) gene set. We note that negative t-statistics means lower expression in cancer compared with normal. b Heatmaps depicting the dynamics of gene expression changes of the tissuespecific TFs expressed in the normal tissue. t-statistics of differential expression (t(DEG)), are shown between hESCs and normal tissue (the left-most colour heatmap in each panel) and between normal tissue and various cancer types (the right heatmap in each panel), as indicated. We note that the heatmap to the very left in each panel is always red, indicating the overexpression of these TFs in foetal/adult normal tissue compared with hESCs. The heatmap representing the t-statistics of differential expression between normal tissue and the corresponding cancer types are shown to the left of the vertical black line, whereas those for the other unrelated cancer types are shown to the right. There is generally more green (i.e. underexpression) in the cancer types matching the tissue types compared with the other cancer types, in agreement with the data shown in a

\section{Promoter hypermethylation, and not CNV loss or mutation, associates most strongly with silencing of bivalent/PRC2-marked TFs in cancer}

We next asked which type of molecular alteration associates most strongly with silencing of bivalently/PRC2marked TFs in cancer. For this analysis, we considered all TFs overexpressed in a given normal tissue type (compared with hESCs) and underexpressed in cancer (compared with its respective normal tissue), without the requirement that they be overexpressed in only one normal tissue type. We obtained CNV, somatic mutation as well as DNAm data for all genes and for all cancer types considered previously ("Methods"). Depicting the copy number and DNAm changes of these silenced TFs between cancers and their corresponding normal samples revealed a striking difference between DNAm and CNV (Fig. 3; Additional file 1: Figures S5-S10). Whereas at the genomic copy number level we did not observe a preference for these TFs to undergo copy number loss, at the level of DNA methylation there was a clear skew towards increased promoter DNAm (Fig. 3; Additional file 1: Figures S5-S10).

In order to assess the statistical and biological significance of these observations, we next compared the degree of molecular alteration of the silenced TFs with that of all genes underexpressed in the given cancer type, as well as to a randomly chosen set of genes, a procedure which adjusts for the differential sensitivity of the different molecular assays. We observed that average genomic loss levels of the silenced TFs were generally not significantly higher than that of underexpressed genes or that of a randomly chosen set of genes (Fig. 4; Additional file 1: Figure S11). Likewise, the average frequency of inactivating mutations of these TFs across cancers was generally not higher compared with underexpressed genes or randomly selected genes (Fig. 4; Additional file 1: Figure S11). In contrast, differential promoter methylation statistics of the silenced TFs were generally significantly higher compared with those of underexpressed or randomly chosen genes (Fig. 4; Additional file 1: Figure S11). In general, for each cancer type there were more TFs and tumours with significant positive differential methylation statistics than the corresponding expected number had the genes been drawn from the set of all cancer underexpressed genes (Additional file 1: Figure S12). This result was also evident if significance in a tumour is defined by a TF exhibiting a promoter DNAm increase of at least $30 \%$ compared with the average over normal samples (Additional file 1: Figure S13). Using a meta-analysis over all cancer types, it was only for the case of promoter hypermethylation that we observed a significantly higher level of alteration at the silenced TFs compared with all underexpressed genes (Table $1 ; P<10^{-8}$ for promoter hypermethylation, $P=0.98$ for $\mathrm{CNV}$ loss and $P=0.47$ for mutation, combined Fisher test). We note that if we compared all underexpressed genes in a given cancer type to a randomly selected set of genes, then all molecular categories were significant, consistent with the view that all molecular events, be it promoter hypermethylation, CNV loss or inactivating mutation, are associated with underexpression in cancer (Additional file 1: Figure S14). In summary, the data shown in Fig. 4 and Table 1 suggest that promoter hypermethylation is the more likely mechanism associated with in-cis TF silencing in cancer.

Next, we decided to extend the previous analysis to the single-sample level in order to investigate the detailed pattern of promoter methylation and $\mathrm{CNV}$ within individual tumours. We first considered for each TF in each cancer type those tumours which exhibited significant underexpression relative to the respective normal tissue ("Methods"). For each TF and across all tumours exhibiting underexpression of this TF, we then counted the fraction of tumours exhibiting genomic loss of the $\mathrm{TF}$, as well as the fraction of tumours exhibiting 


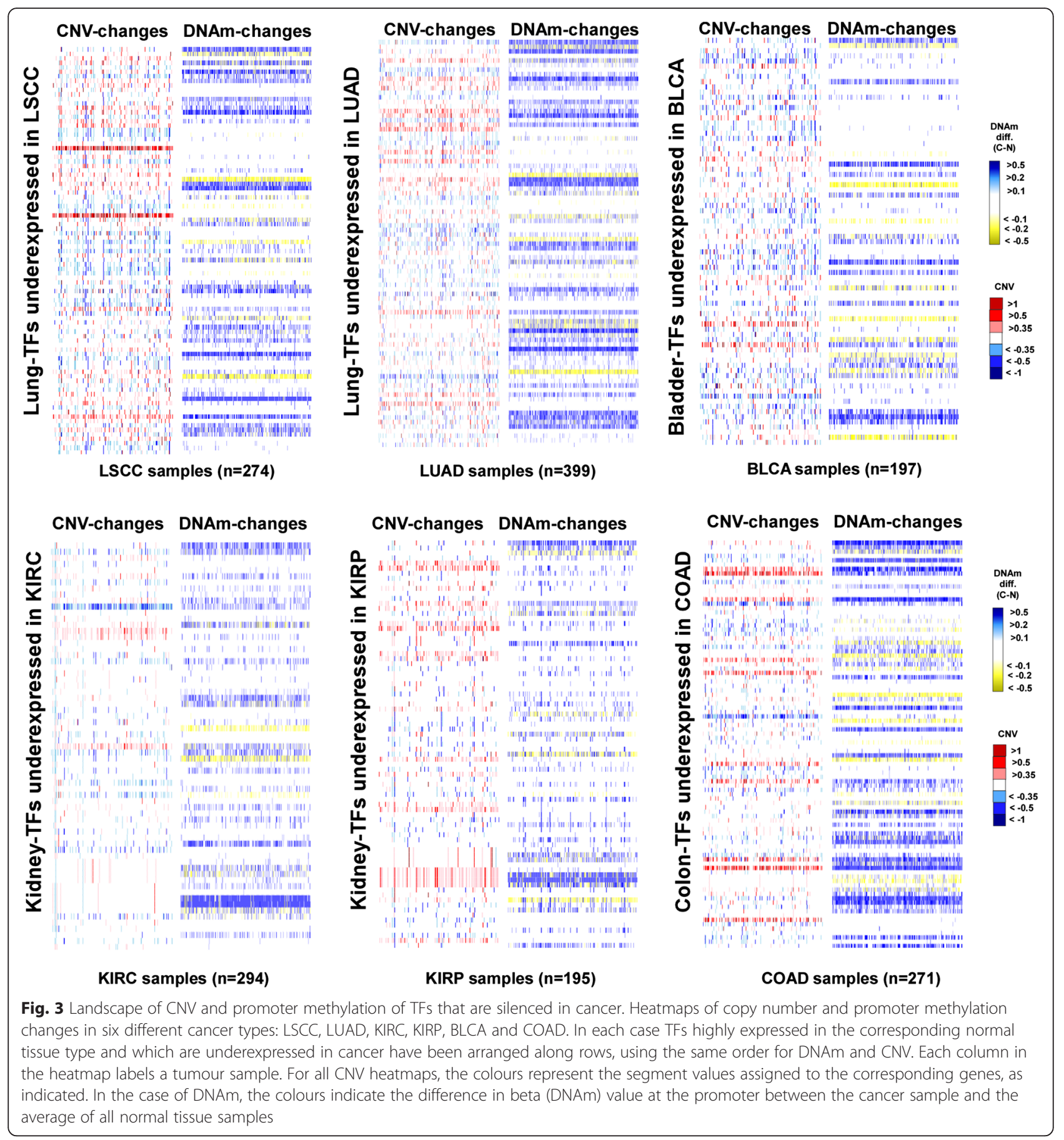

hypermethylation of the TF's promoter ("Methods"). In general, this revealed that promoter hypermethylation events could account for a higher fraction of cancers exhibiting underexpression of the corresponding TF compared with genomic loss (Fig. 5a). For instance, in LSCC we observed four TFs (HOXA4, HOXA5, TAL1, ZNF132) undergoing promoter hypermethylation in at least $50 \%$ of the LSCC tumour samples where these TFs were underexpressed. In contrast, no TF was observed to undergo CNV loss at a frequency of over $50 \%$ in the same cancers (Fig. 5a). A similar observation was evident for LUAD (Fig. 5a). In the case of KIRP we observed six TFs exhibiting promoter hypermethylation at over $20 \%$ of the tumours with underexpression of the TF, in contrast to no TF exhibiting CNV loss at that frequency or higher (Fig. 5a). This pattern of more frequent promoter hypermethylation than CNV loss was also evident for BLCA and COAD (Fig. 5a). 

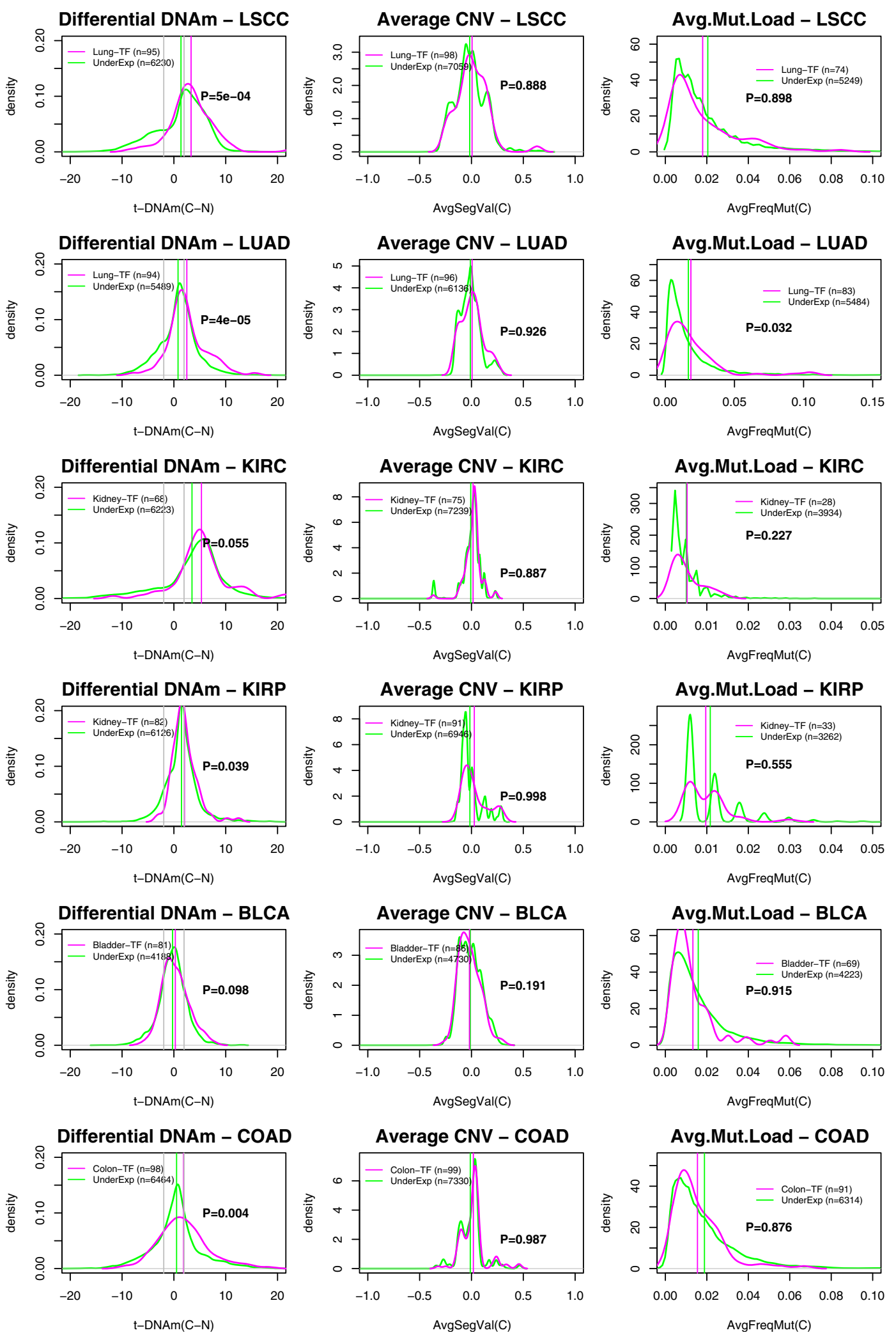

Fig. 4 (See legend on next page.) 
(See figure on previous page.)

Fig. 4 Transcription factors expressed in normal tissue and silenced in cancer predominantly exhibit promoter hypermethylation and not genomic loss or inactivating mutation. Left panels: density plots of t-statistics of differential DNAm between cancer and normal tissue $(x-a x i s, t(C-N))$ of the tissue-specific cancer-silenced TFs (magenta lines) compared with the corresponding density distribution of all genes underexpressed in cancer (green lines). Density plots are shown for six cancer types: LSCC, LUAD, KIRC, KIRP, BLCA and COAD. $P$ values are from a Wilcoxon rank sum test. The vertical magenta and green lines denote the average levels. The grey vertical lines in the DNAm plot indicate $P=0.05$. Middle panels: as above but for the average CNV segment values of the TFs (magenta lines) and all underexpressed genes (green lines). Right panels: as above but for the frequency of inactivating mutation of the TFs (magenta lines) and all underexpressed genes (green lines)

\section{Some silenced bivalent/PRC2-marked TFs exhibit patterns of mutual exclusivity between promoter hypermethylation and CNV loss}

Interestingly, we observed that many TFs exhibiting a higher frequency of CNV loss in cancer did not show appreciable promoter DNAm increases in any of the tumour samples, suggesting that some TFs are more intrinsically prone to genomic loss (Fig. 5a). Indeed, broadly speaking, there were three types of silenced TFs in each cancer type (Fig. 5b): those predominantly exhibiting promoter hypermethylation but with relatively few CNV losses (e.g. FOXF1 in LUAD, HAND2 in COAD), those exhibiting frequent $\mathrm{CNV}$ loss but not many DNAm changes (e.g. NR2F1 in LSCC, FOXO3 in LUAD, SETBP1 in COAD) and a third class of TFs which exhibited both CNV loss and promoter hypermethylation (e.g. ZNF132 in LUAD, HIC1 in COAD).

To investigate if there is any evidence for mutual exclusivity between promoter hypermethylation and CNV loss, we next compared the frequency of TF promoter hypermethylation between the top and lowest tertiles of TFs ranked by CNV loss frequency. This revealed a higher frequency of hypermethylation for those TFs undergoing the least $\mathrm{CNV}$

Table 1 Silenced TFs in cancer undergo preferential promoter hypermethylation in comparison with all cancer underexpressed genes

\begin{tabular}{llllll}
\hline & $\mathrm{nTF}$ & $\mathrm{nU}$ & $P$ (DNAm) & $P(\mathrm{CNV})$ & $P$ (Mutation) \\
\hline LSCC & 95 & 7697 & 0.0005 & 0.888 & 0.898 \\
LUAD & 94 & 6673 & 0.00004 & 0.926 & 0.032 \\
KIRC & 68 & 7814 & 0.055 & 0.887 & 0.227 \\
KIRP & 82 & 7465 & 0.039 & 0.998 & 0.555 \\
BLCA & 81 & 5097 & 0.098 & 0.191 & 0.915 \\
COAD & 98 & 7934 & 0.004 & 0.987 & 0.876 \\
\multicolumn{2}{l}{ Combined Fisher test } & & $<1 \mathrm{e}-8$ & 0.984 & 0.471 \\
\hline
\end{tabular}

The first six rows label the TCGA cancer type. Columns label the number of TFs expressed in the normal tissue and underexpressed in cancer (nTF), the number of all genes underexpressed in cancer $(n U)$, the $P$ value from a Wilcoxon rank sum test assessing whether promoter hypermethylation at the TFs is more frequent than for all underexpressed genes with DNAm information (DNAm), the $P$ value from a Wilcoxon rank sum test assessing whether CNV loss of the TFs is more frequent than for all underexpressed genes with CNV information (CNV) and the $P$ value from a Wilcoxon rank sum test assessing whether inactivating mutation of the TFs is more frequent than for all underexpressed genes with mutational information (Mutation). The last row lists $P$ values of a meta-analysis over all cancer types using a combined Fisher test losses (Additional file 1: Figure S15a; combined Fisher test $P=0.002$ ), consistent with the observed "L" type shapes of the scatterplots (Fig. 5a). The reverse analysis, comparing the frequency of $\mathrm{CNV}$ loss between the top and lowest tertiles defined according to the frequency of hypermethylation, also revealed a consistent pattern of mutual exclusivity (Additional file 1: Figure S15b; combined Fisher test $P=0.004)$.

Focusing on TFs undergoing both CNV loss and promoter hypermethylation (at least $1 \%$ frequency for both types of alteration) revealed only a few (EBF1 in LSCC, LYL1 in LUAD, ZNF287 in BLCA and HIC1 in COAD) which did so in a mutually exclusive fashion, in the sense of exhibiting higher levels of hypermethylation in tumours with no CNV loss of the given TF, compared with tumours with CNV loss, although this was only evident if the previous threshold for calling significant promoter hypermethylation (i.e. 0.3) was relaxed to a value of 0.1 (Additional file 1: Figure S16).

\section{Bivalent/PRC2-marked TFs silenced in multiple cancer types are more likely to share aberrant promoter hypermethylation}

Next, we asked if the mechanism associated with silenced TFs is similar between cancer types. For this analysis, we focused on TFs that were commonly silenced across cancer types. As expected, LSCC and LUAD shared a strong overlap of 80 TFs $(\sim 88 \%)$ silenced in both cancer types, whilst the smallest overlap was between BLCA and KIRC (18 TFs). Frequencies of promoter hypermethylation of commonly silenced TFs were highly correlated between every pair of cancer types (average $\mathrm{R}^{2}$ value was 0.39; Additional file 1: Figure S17). In contrast, correlations were significantly lower in the case of CNV loss (average $\mathrm{R}^{2}$ value was 0.23 , Wilcoxon rank sum paired test $P=0.005$; Additional file 1: Figure S18). This suggests that TFs silenced in multiple cancer types are more likely to be associated with promoter DNA hypermethylation than with in-cis CNV loss.

\section{Discussion}

Although impairment of differentiation is a well known cancer hallmark, only a few concrete examples of TF inactivation have been shown to block differentiation and predispose to epithelial cancer $[8,9]$. Since the experimental 


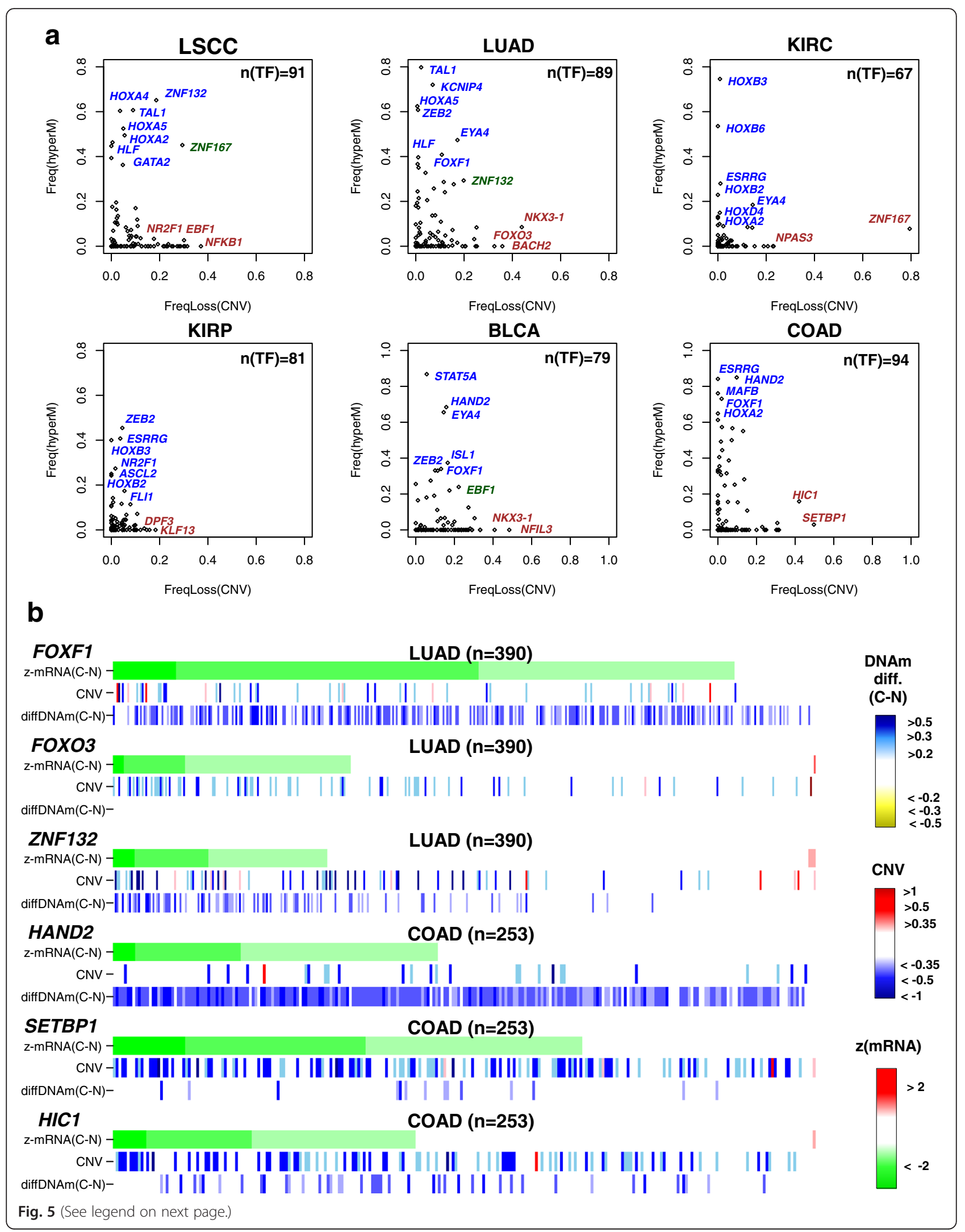


(See figure on previous page.)

Fig. 5 Cancer-silenced TFs exhibiting different propensities to undergo promoter DNA methylation or genomic loss in cancer. a Scatterplots of the frequency of genomic loss ( $x$-axis) against promoter hypermethylation ( $y$-axis) in cancer, as estimated over tumours exhibiting underexpression of the given TF. Each data point in the scatterplots represents one silenced TF. Some of the TFs exhibiting more propensity to undergo promoter DNAm than CNV loss are shown in blue, some TFs exhibiting less propensity to undergo promoter DNAm than CNV loss are shown in brown, and in green we highlight some TFs exhibiting both frequent CNV loss and promoter hypermethylation. $\mathbf{b}$ Heatmap representations of mRNA expression change (z-statistics of mRNA expression change), CNV and DNAm change (difference in beta-value between cancer and all normals) for a number of silenced TFs exhibiting different propensities for promoter hypermethylation and CNV loss in two different cancer types (LUAD and COAD), as indicated. Tumour samples are sorted in decreasing order of underexpression in cancer

identification of TFs necessary for tissue specification is cumbersome, we here took an in silico approach, comparing mRNA expression levels of a relevant subset of TFs (bivalently and PRC2-marked) between hESCs and normal foetal/adult tissue in order to identify TFs which become strongly overexpressed upon differentiation. We hypothesized that if blocks in differentiation constitute a key process contributing to carcinogenesis, these highly expressed TFs would be frequently silenced in cancer and they would do so preferentially in comparison with other non-housekeeping genes which are highly expressed in the same tissue. Using six different cancer types, we were able to confirm that TFs overexpressed in a normal tissue type relative to a hESC ground state are preferentially silenced in the corresponding tumour type. These TFs likely represent tumour suppressors. Our second main contribution is the demonstration that silencing of these TFs is associated mainly with promoter hypermethylation and not with in-cis genomic loss or mutation. Importantly, for many TFs, promoter hypermethylation could account for the largest fractions of tumours exhibiting underexpression of that TF. Indeed, whereas CNV loss and inactivation mutations are known to affect tumour suppressors, the frequencies of these events across tumours of a given cancer type are generally quite low, making it difficult to identify novel cancer driver genes [59]. In contrast, promoter hypermethylation at specific TFs is a much more frequent event, supporting a role for epigenetic-mediated silencing in the suppression of key tumour suppressors [60]. However, we also observed silenced TFs which were only prone to CNV loss with no observed promoter hypermethylation across tumours. In addition, we also identified a few examples of silenced TFs exhibiting both CNV loss and promoter hypermethylation in a mutually exclusive fashion.

While these novel insights support the view that promoter hypermethylation of lineage-specifying TFs could be a key step in carcinogenesis, it is equally important to point out limitations in our analysis. First of all, it is important to stress that the observed correlations between promoter DNAm and underexpression are only associative. Demonstrating that the observed promoter hypermethylation causes TF underexpression is beyond the scope of this study. Moreover, we can't exclude the possibility that inactivation of an upstream TF, through genomic loss or mutation, underlies the loss of binding and hence increased DNAm at the promoters of the observed TFs. Indeed, several studies have shown how hypermethylation at both promoters and distal regulatory elements such as enhancers can result from deletion of specific TFs [61]. Also, the important role of DNAm alterations at super-enhancers and associated DNAm and mRNA expression changes at linked gene promoters in cancer has recently been noted [62]. Thus, our data can't distinguish between a causative model, in which promoter hypermethylation causes the observed underexpression of the TFs, from an effects model, in which the observed hypermethylation and silencing is the consequence of an upstream TF inactivation event, be this a CNV loss, inactivating mutation, promoter methylation or increased methylation at an enhancer. The associative statistical analysis presented here suggests, however, that, probabilistically, promoter hypermethylation of a TF is a more likely mechanism than CNV loss or an inactivating mutation.

A second limitation of our analysis is that we did not consider the role of non-coding RNAs, in particular that of microRNAs (miRNAs). In common with TFs, miRNAs play an important role in development and cellular differentiation, with many playing a tumour-suppressive role in cancer $[63,64]$. Moreover, it has recently been noted that bivalently marked miRNA promoters are also frequently hypermethylated in cancer, with many of these also exhibiting underexpression [65]. It will be interesting, therefore, to explore if miRNAs highly expressed in a given tissue type also exhibit preferential downregulation in the corresponding cancer type and whether, for this particular subset of downregulated miRNAs, promoter hypermethylation is also the main associative mechanism. Likewise, in this study we did not consider the important role of histone modifications, which we know are altered in cancer and which could also result in epigenetic silencing of key TFs, as observed, for instance, in the case of HNF4A in liver cancer, where the reduced expression has been attributed to a loss of H3K4me3 [8, 66]. Unfortunately, histone modification data for the matched TCGA samples considered here are not available. In future, however, it will be important to include ChIP-Seq profiles for 
all major regulatory histone marks in these comparative analyses.

A third caveat in our analysis is that the inferred underexpression of TFs in cancer was done by comparison with a normal reference defined by normal tissue that is found adjacent to the tumour specimen. This normaladjacent tissue may already contain age-associated epigenetic field defects [67], which may reduce the sensitivity to detect silencing events in cancer. For instance, GATA4 is a well known differentiation factor for a number of different tissue types, including colon tissue [39]. Although we did observe GATA4 to be overexpressed in foetal colon tissue compared with hESCs, its level of mRNA expression in the normal colon tissue adjacent to colorectal cancer samples was surprisingly low, which is why we did not see further underexpression of this TF in colon cancer. A potential explanation for this is that GATA4 is already gradually silenced in aged colon tissue as a result of age-associated promoter hypermethylation [13], with the aggravated hypermethylation in cancer not causing any further change in gene expression. Direct comparison with a purified age-matched sample representing the cell of origin could overcome some of these limitations. A related caveat in our analysis is cellular heterogeneity, as it is possible that the cell of origin of the cancer is underrepresented in the normal tissue, confounding the differential expression analysis, although this is less likely to be the case for normal tissue found adjacent to the cancer.

Another limitation is the restriction to four tissue types (lung, kidney, bladder and colon). This restriction merely reflects the availability of mRNA expression data in the original SCM2 compendium which simultaneously profiled hESCs and primary differentiated cells for a number of different tissue types. Given that studyspecific batch effects are notorious in gene expression data [68], the requirement that expression profiles from hESCs and differentiated tissue come from the same study is critical. Analysis of a more comprehensive compendium of hESC and differentiated primary samples using RNA-Seq data will be needed to assess whether the findings reported here generalize to other tissue types. However, in spite of only analyzing four normal tissues and six cancer types, our results are highly statistically significant when interpreted in the context of a meta-analysis (see e.g. Table 1).

Finally, we stress that most of the analyses presented here were performed on TFs expressed in a normal tissue type, regardless of their expression levels in other normal tissues. Although this entails a much more liberal definition of "tissue specificity", it is also the most biologically meaningful one to consider. For instance, as remarked earlier, HNF4A is a TF which is needed for liver specification, silencing of it leading to liver cancer
[8], yet it is also expressed in other tissue types such as kidney and stomach [35]. Hence, TFs expressed in multiple normal tissue types can be as important to the development of a specific cancer-type than TFs which are expressed only in the corresponding normal tissue type. In line with this, we have seen that a considerable number of TFs are overexpressed in many different tissue types and also seen to be silenced in common between cancer types. For instance, between lung, kidney, bladder and colon tissue, ten TFs (CASZ1, NR3C2, THRA, SETBP1, SMARCA2, MEIS2, NFIC, PURA, KLF13, TCF21) were commonly overexpressed in all these tissues compared with hESCs and also commonly silenced in LSCC, LUAD, KIRC, KIRP, BLCA and COAD compared with their respective normal tissues. This list includes known tumour suppressors such as the nuclear receptor NR3C2 [69], the helix-loop-helix transcription factor TCF21 [70], and SMARCA2 (also known as BRM), a member of the SNF/ SWI chromatin remodelling complex [71-73]. Interestingly, however, the list also includes SETBP1, a TF which has been reported to be oncogenic in myeloid neoplasms [74, 75], highlighting the need to explore a potential tumour suppressive role of this TF in the context of epithelial cancer.

\section{Conclusions}

The data presented here support the view that bivalently and PRC2-marked TFs expressed in a given normal tissue are more likely to undergo silencing in the corresponding cancer type compared with other non-housekeeping genes that are highly expressed in the same normal tissue. This suggests that putative differentiation blocks arising as a result of their inactivation are strongly selected for during carcinogenesis. Importantly, our data suggest that the silencing of these TFs in cancer is predominantly associated with promoter hypermethylation.

\section{Additional file}

Additional file 1: This document contains all Supplementary Tables and all Supplementary Figures, plus their associated legends/captions. (PDF $3658 \mathrm{~kb}$ )

\section{Acknowledgements}

The authors wish to thank the Chinese Academy of Sciences, Shanghai Institute for Biological Sciences and the Max-Planck Society. The results shown here are in part based upon data generated by TCGA Research Network, to which we are most grateful.

\section{Funding}

This study was supported by NSFC (National Science Foundation of China) grant numbers 31571359 and 31401120 and by a Royal Society Newton Advanced Fellowship (NAF award number 164914).

Availability of data and materials

The datasets analysed during the current study are available from The Cancer Genome Atlas Data portal (http://www.cbioportal.org). 


\section{Authors' contributions}

AET conceived of the study, performed statistical analyses and wrote the manuscript. SCZ helped with statistical analyses. YZ helped with preprocessing of data. AF, MW and SB contributed valuable feedback. All authors read and approved the final manuscript.

\section{Competing interests}

The authors declare that they have no competing interests.

\section{Author details}

'CAS Key Laboratory of Computational Biology, CAS-MPG Partner Institute for Computational Biology, Chinese Academy of Sciences, Shanghai Institute for Biological Sciences, 320 Yue Yang Road, Shanghai 200031, China. ${ }^{2}$ Statistical Cancer Genomics, UCL Cancer Institute, University College London, Paul O'Gorman Building, 72 Huntley Street, London WC1E 6BT, UK. ${ }^{3}$ Department of Women's Cancer, University College London, 74 Huntley Street, London WC1E 6BT, UK. ${ }^{4}$ Medical Genomics, UCL Cancer Institute, University College London, Paul O'Gorman Building, 72 Huntley Street, London WC1E 6BT, UK.

Received: 6 May 2016 Accepted: 5 August 2016

Published online: 25 August 2016

\section{References}

1. Heinaniemi M, Nykter M, Kramer R, Wienecke-Baldacchino A, Sinkkonen L, Zhou JX, Kreisberg R, Kauffman SA, Huang S, Shmulevich I. Gene-pair expression signatures reveal lineage control. Nat Methods. 2013;10:577-83.

2. Yamanaka S, Blau HM. Nuclear reprogramming to a pluripotent state by three approaches. Nature. 2010;465:704-12.

3. Feinberg AP, Ohlsson R, Henikoff $S$. The epigenetic progenitor origin of human cancer. Nat Rev Genet. 2006;7:21-33.

4. Widschwendter M, Fiegl H, Egle D, Mueller-Holzner E, Spizzo G, Marth C, Weisenberger DJ, Campan M, Young J, Jacobs I, Laird PW. Epigenetic stem cell signature in cancer. Nat Genet. 2007;39:157-8.

5. Ohm JE, McGarvey KM, Yu X, Cheng L, Schuebel KE, Cope L, Mohammad HP, Chen W, Daniel VC, Yu W, et al. A stem cell-like chromatin pattern may predispose tumor suppressor genes to DNA hypermethylation and heritable silencing. Nat Genet. 2007;39:237-42.

6. Baylin SB, Ohm JE. Epigenetic gene silencing in cancer-a mechanism for early oncogenic pathway addiction? Nat Rev Cancer. 2006;6:107-16.

7. Jones PA, Baylin SB. The fundamental role of epigenetic events in cancer. Nat Rev Genet. 2002;3:415-28.

8. Saha SK, Parachoniak CA, Ghanta KS, Fitamant J, Ross KN, Najem MS, Gurumurthy S, Akbay EA, Sia D, Cornella H, et al. Mutant IDH inhibits HNF4alpha to block hepatocyte differentiation and promote biliary cancer. Nature. 2014:513:110-4.

9. Lu C, Ward PS, Kapoor GS, Rohle D, Turcan S, Abdel-Wahab O, Edwards CR, Khanin R, Figueroa ME, Melnick A, et al. IDH mutation impairs histone demethylation and results in a block to cell differentiation. Nature. 2012;483: 474-8.

10. Bernstein BE, Mikkelsen TS, Xie X, Kamal M, Huebert DJ, Cuff J, Fry B, Meissner A, Wernig M, Plath $K$, et al. A bivalent chromatin structure marks key developmental genes in embryonic stem cells. Cell. 2006;125:315-26.

11. Lee TI, Jenner RG, Boyer LA, Guenther MG, Levine SS, Kumar RM, Chevalier B, Johnstone SE, Cole MF, Isono K, et al. Control of developmental regulators by Polycomb in human embryonic stem cells. Cell. 2006;125:301-13.

12. Schlesinger $Y$, Straussman R, Keshet I, Farkash S, Hecht M, Zimmerman J, Eden E, Yakhini Z, Ben-Shushan E, Reubinoff BE, et al. Polycomb-mediated methylation on Lys27 of histone $\mathrm{H} 3$ pre-marks genes for de novo methylation in cancer. Nat Genet. 2007;39:232-6.

13. Teschendorff AE, Menon U, Gentry-Maharaj A, Ramus SJ, Weisenberger DJ, Shen H, Campan M, Noushmehr H, Bell CG, Maxwell AP, et al. Agedependent DNA methylation of genes that are suppressed in stem cells is a hallmark of cancer. Genome Res. 2010;20:440-6.

14. Rakyan VK, Down TA, Maslau S, Andrew T, Yang TP, Beyan H, Whittaker P, McCann OT, Finer S, Valdes AM, et al. Human aging-associated DNA hypermethylation occurs preferentially at bivalent chromatin domains. Genome Res. 2010:20:434-9.

15. Hannum G, Guinney J, Zhao L, Zhang L, Hughes G, Sadda S, Klotzle B, Bibikova M, Fan JB, Gao Y, et al. Genome-wide methylation profiles reveal quantitative views of human aging rates. Mol Cell. 2013;49:359-67.
16. Kulis M, Merkel A, Heath S, Queiros AC, Schuyler RP, Castellano G, Beekman R, Raineri E, Esteve A, Clot G, et al. Whole-genome fingerprint of the DNA methylome during human B cell differentiation. Nat Genet. 2015;47:746-56.

17. Sproul D, Kitchen RR, Nestor CE, Dixon JM, Sims AH, Harrison DJ, Ramsahoye BH, Meehan RR. Tissue of origin determines cancer-associated CpG island promoter hypermethylation patterns. Genome Biol. 2012;13:R84.

18. Nejman D, Straussman R, Steinfeld I, Ruvolo M, Roberts D, Yakhini Z, Cedar H. Molecular rules governing de novo methylation in cancer. Cancer Res. 2014;74: 1475-83.

19. Nazor KL, Altun G, Lynch C, Tran H, Harness JV, Slavin I, Garitaonandia I, Muller FJ, Wang YC, Boscolo FS, et al. Recurrent variations in DNA methylation in human pluripotent stem cells and their differentiated derivatives. Cell Stem Cell. 2012;10:620-34.

20. Muller FJ, Laurent LC, Kostka D, Ulitsky I, Williams R, Lu C, Park IH, Rao MS, Shamir R, Schwartz PH, et al. Regulatory networks define phenotypic classes of human stem cell lines. Nature. 2008;455:401-5.

21. Smyth GK. Linear models and empirical bayes methods for assessing differential expression in microarray experiments. Stat Appl Genet Mol Biol. 2004:3:Article3.

22. Wettenhall JM, Smyth GK. limmaGUI: a graphical user interface for linear modeling of microarray data. Bioinformatics. 2004:20:3705-6.

23. Cancer Genome Atlas Research N. Comprehensive molecular profiling of lung adenocarcinoma. Nature. 2014;511:543-50.

24. Cancer Genome Atlas Research N. Comprehensive genomic characterization of squamous cell lung cancers. Nature. 2012;489:519-25.

25. Cancer Genome Atlas Research N. Comprehensive molecular characterization of clear cell renal cell carcinoma. Nature. 2013;499:43-9.

26. Cancer Genome Atlas Research N, Linehan WM, Spellman PT, Ricketts CJ, Creighton CJ, Fei SS, Davis C, Wheeler DA, Murray BA, Schmidt L, et al. Comprehensive molecular characterization of papillary renal-cell carcinoma. N Engl J Med. 2016;374:135-45

27. Cancer Genome Atlas Research N. Comprehensive molecular characterization of urothelial bladder carcinoma. Nature. 2014;507:315-22.

28. Muzny DM, Bainbridge MN, Chang K, Dinh HH, Drummond JA, Fowler G, Kovar CL, Lewis LR, Morgan MB, Newsham IF, et al. Comprehensive molecular characterization of human colon and rectal cancer. Nature. 2012:487:330-7.

29. Cancer Genome Atlas Research N. Comprehensive molecular characterization of gastric adenocarcinoma. Nature. 2014:513:202-9.

30. Teschendorff AE, Marabita F, Lechner M, Bartlett T, Tegner J, Gomez-Cabrero D, Beck S. A beta-mixture quantile normalization method for correcting probe design bias in Illumina Infinium 450 k DNA methylation data. Bioinformatics. 2013;29:189-96.

31. Teschendorff A. Computational and Statistical Epigenomics. Dordrecht: Springer; 2015.

32. Jiao $Y$, Widschwendter M, Teschendorff AE. A systems-level integrative framework for genome-wide DNA methylation and gene expression data identifies differential gene expression modules under epigenetic control. Bioinformatics. 2014;30(16):2360-6.

33. Eisenberg E, Levanon EY. Human housekeeping genes, revisited. Trends Genet. 2013;29:569-74

34. Ye DZ, Kaestner KH. Foxa1 and Foxa2 control the differentiation of goblet and enteroendocrine L- and D-cells in mice. Gastroenterology. 2009;137: 2052-62.

35. Uhlen M, Fagerberg L, Hallstrom BM, Lindskog C, Oksvold P, Mardinoglu A, Sivertsson A, Kampf C, Sjostedt E, Asplund A, et al. Proteomics. Tissue-based map of the human proteome. Science. 2015;347:1260419.

36. Cereghini S. Liver-enriched transcription factors and hepatocyte differentiation. FASEB J. 1996;10:267-82.

37. Walker EM, Thompson CA, Kohlnhofer BM, Faber ML, Battle MA Characterization of the developing small intestine in the absence of either GATA4 or GATA6. BMC Res Notes. 2014;7:902.

38. Walker EM, Thompson CA, Battle MA. GATA4 and GATA6 regulate intestinal epithelial cytodifferentiation during development. Dev Biol. 2014;392:283-94.

39. Akiyama $Y$, Watkins $N$, Suzuki $H$, Jair KW, van Engeland M, Esteller M, Sakai $H_{\text {, }}$ Ren CY, Yuasa Y, Herman JG, Baylin SB. GATA-4 and GATA-5 transcription factor genes and potential downstream antitumor target genes are epigenetically silenced in colorectal and gastric cancer. Mol Cell Biol. 2003;23:8429-39.

40. Zhou P, He A, Pu WT. Regulation of GATA4 transcriptional activity in cardiovascular development and disease. Curr Top Dev Biol. 2012;100:143-69.

41. Xuan S, Sussel L. GATA4 and GATA6 regulate pancreatic endoderm identity through inhibition of hedgehog signaling. Development. 2016;143:780-6. 
42. Borok MJ, Papaioannou VE, Sussel L. Unique functions of Gata4 in mouse liver induction and heart development. Dev Biol. 2016;410:213-22.

43. Agnihotri S, Wolf A, Munoz DM, Smith CJ, Gajadhar A, Restrepo A, Clarke ID, Fuller GN, Kesari S, Dirks PB, et al. A GATA4-regulated tumor suppressor network represses formation of malignant human astrocytomas. J Exp Med. 2011;208:689-702.

44. Jimenez FR, Lewis JB, Belgique ST, Wood TT, Reynolds PR. Developmental lung expression and transcriptional regulation of claudin- 6 by TTF-1, Gata- 6 , and FoxA2. Respir Res. 2014;15:70.

45. Arora R, Metzger RJ, Papaioannou VE. Multiple roles and interactions of Tbx4 and Tbx5 in development of the respiratory system. PLoS Genet. 2012;8: e1002866.

46. Batra H, Antony VB. The pleural mesothelium in development and disease. Front Physiol. 2014:5:284

47. Liu WB, Jiang X, Han F, Li YH, Chen HQ, Liu Y, Cao J, Liu JY. LHX6 acts as a novel potential tumour suppressor with epigenetic inactivation in lung cancer. Cell Death Dis. 2013;4:e882.

48. Yu MJ, Miller RL, Uawithya P, Rinschen MM, Khositseth S, Braucht DW, Chou CL, Pisitkun T, Nelson RD, Knepper MA. Systems-level analysis of cell-specific AQP2 gene expression in renal collecting duct. Proc Natl Acad Sci U S A. 2009:106:2441-6.

49. Berry R, Harewood L, Pei L, Fisher M, Brownstein D, Ross A, Alaynick WA, Moss J, Hastie ND, Hohenstein P, et al. Esrrg functions in early branch generation of the ureteric bud and is essential for normal development of the renal papilla. Hum Mol Genet. 2011;20:917-26.

50. Lam AQ, Freedman BS, Morizane R, Lerou PH, Valerius MT, Bonventre JV. Rapid and efficient differentiation of human pluripotent stem cells into intermediate mesoderm that forms tubules expressing kidney proximal tubular markers. J Am Soc Nephrol. 2014;25:1211-25.

51. Gallegos TF, Kouznetsova V, Kudlicka K, Sweeney DE, Bush KT, Willert K, Farquhar MG, Nigam SK. A protein kinase A and Wnt-dependent network regulating an intermediate stage in epithelial tubulogenesis during kidney development. Dev Biol. 2012;364:11-21.

52. DeGraff DJ, Cates JM, Mauney JR, Clark PE, Matusik RJ, Adam RM. When urothelial differentiation pathways go wrong: implications for bladder cancer development and progression. Urol Oncol. 2013;31:802-11.

53. Park MJ, Kim HY, Kim K, Cheong J. Homeodomain transcription factor CDX1 is required for the transcriptional induction of PPARgamma in intestinal cell differentiation. FEBS Lett. 2009;583:29-35.

54. Soubeyran P, Andre F, Lissitzky JC, Mallo GV, Moucadel V, Roccabianca M, Rechreche H, Marvaldi J, Dikic I, Dagorn JC, lovanna JL. Cdx1 promotes differentiation in a rat intestinal epithelial cell line. Gastroenterology. 1999; 117:1326-38.

55. Crissey MA, Guo RJ, Funakoshi S, Kong J, Liu J, Lynch JP. Cdx2 levels modulate intestinal epithelium maturity and Paneth cell development. Gastroenterology. 2011;140:517-28. e518.

56. Gao N, White P, Kaestner KH. Establishment of intestinal identity and epithelial-mesenchymal signaling by Cdx2. Dev Cell. 2009;16:588-99.

57. Desai S, Loomis Z, Pugh-Bernard A, Schrunk J, Doyle MJ, Minic A, McCoy E, Sussel L. Nkx2.2 regulates cell fate choice in the enteroendocrine cell lineages of the intestine. Dev Biol. 2008;313:58-66.

58. Schonhoff SE, Giel-Moloney M, Leiter AB. Minireview: Development and differentiation of gut endocrine cells. Endocrinology. 2004;145:2639-44.

59. Vogelstein B, Papadopoulos N, Velculescu VE, Zhou S, Diaz Jr LA, Kinzler KW. Cancer genome landscapes. Science. 2013:339:1546-58.

60. Jones A, Teschendorff AE, Li Q, Hayward JD, Kannan A, Mould T, West J, Zikan M, Cibula D, Fiegl H, et al. Role of DNA methylation and epigenetic silencing of HAND2 in endometrial cancer development. PLoS Med. 2013; 10:e1001551.

61. Feldmann A, Ivanek R, Murr R, Gaidatzis D, Burger L, Schubeler D. Transcription factor occupancy can mediate active turnover of DNA methylation at regulatory regions. PLoS Genet. 2013;9:e1003994.

62. Heyn H, Vidal E, Ferreira HJ, Vizoso M, Sayols S, Gomez A, Moran S, BoqueSastre R, Guil S, Martinez-Cardus A, et al. Epigenomic analysis detects aberrant super-enhancer DNA methylation in human cancer. Genome Biol. 2016;17:11.

63. Lujambio A, Calin GA, Villanueva A, Ropero S, Sanchez-Cespedes M, Blanco D, Montuenga LM, Rossi S, Nicoloso MS, Faller WJ, et al. A microRNA DNA methylation signature for human cancer metastasis. Proc Natl Acad Sci U S A. 2008;105:13556-61.

64. Blenkiron C, Goldstein LD, Thorne NP, Spiteri I, Chin SF, Dunning MJ, Barbosa-Morais NL, Teschendorff AE, Green AR, Ellis IO, et al. MicroRNA expression profiling of human breast cancer identifies new markers of tumor subtype. Genome Biol. 2007;8:R214.

65. Iliou MS, Lujambio A, Portela A, Brustle O, Koch P, Andersson-Vincent PH, Sundstrom E, Hovatta O, Esteller M. Bivalent histone modifications in stem cells poise miRNA loci for CpG island hypermethylation in human cancer. Epigenetics. 2011;6(11):1344-53.

66. Saha SK, Parachoniak CA, Bardeesy N. IDH mutations in liver cell plasticity and biliary cancer. Cell Cycle. 2014;13:3176-82

67. Teschendorff AE, Gao Y, Jones A, Ruebner M, Beckmann MW, Wachter DL, Fasching PA, Widschwendter M. DNA methylation outliers in normal breast tissue identify field defects that are enriched in cancer. Nat Commun. 2016;7:10478.

68. Leek JT, Scharpf RB, Bravo HC, Simcha D, Langmead B, Johnson WE, Geman $D$, Baggerly K, Irizarry RA. Tackling the widespread and critical impact of batch effects in high-throughput data. Nat Rev Genet. 2010;11:733-9.

69. Yang S, He P, Wang J, Schetter A, Tang W, Funamizu N, Yanaga K, Uwagawa T, Satoskar AR, Gaedcke J, et al. A Novel MIF Signaling Pathway Drives the Malignant Character of Pancreatic Cancer by Targeting NR3C2. Cancer Res. 2016;76(13):3838-50.

70. Smith LT, Lin M, Brena RM, Lang JC, Schuller DE, Otterson GA, Morrison CD, Smiraglia DJ, Plass C. Epigenetic regulation of the tumor suppressor gene TCF21 on 6q23-q24 in lung and head and neck cancer. Proc Natl Acad Sci U S A. 2006;103:982-7

71. Xia QY, Rao Q, Cheng L, Shen Q, Shi SS, Li L, Liu B, Zhang J, Wang YF, Shi QL et al. Loss of BRM expression is a frequently observed event in poorly differentiated clear cell renal cell carcinoma. Histopathology. 2014:64:847-62.

72. Rao Q, Xia QY, Wang ZY, Li L, Shen Q, Shi SS, Wang X, Liu B, Wang YF, Shi QL, et al. Frequent co-inactivation of the SWI/SNF subunits SMARCB1, SMARCA2 and PBRM1 in malignant rhabdoid tumours. Histopathology. 2015;67:121-9.

73. Kahali B, Gramling SJ, Marquez SB, Thompson K, Lu L, Reisman D. Identifying targets for the restoration and reactivation of BRM. Oncogene. 2014;33:653-64.

74. Piazza R, Valletta S, Winkelmann N, Redaelli S, Spinelli R, Pirola A, Antolini L, Mologni L, Donadoni C, Papaemmanuil E, et al. Recurrent SETBP1 mutations in atypical chronic myeloid leukemia. Nat Genet. 2013;45:18-24.

75. Makishima H, Yoshida K, Nguyen N, Przychodzen B, Sanada M, Okuno Y, $\mathrm{Ng} \mathrm{KP}$, Gudmundsson KO, Vishwakarma BA, Jerez A, et al. Somatic SETBP1 mutations in myeloid malignancies. Nat Genet. 2013;45:942-6.

\section{Submit your next manuscript to BioMed Central and we will help you at every step:}

- We accept pre-submission inquiries

- Our selector tool helps you to find the most relevant journal

- We provide round the clock customer support

- Convenient online submission

- Thorough peer review

- Inclusion in PubMed and all major indexing services

- Maximum visibility for your research

Submit your manuscript at www.biomedcentral.com/submit
Ciomed Central 